\title{
A Pervasive Approach to a Real-Time Intelligent Decision Support System in Intensive Medicine
}

\author{
Filipe Portela, Manuel Filipe Santos, and Marta Vilas-Boas \\ Departamento de Sistemas de Informação, Universidade do Minho \\ Campus de Azurém 4800-058 Guimarães, Portugal \\ $\{\mathrm{cfp}, \mathrm{mfs}, \mathrm{mvb}\} @ \mathrm{dsi}$. uminho.pt
}

\begin{abstract}
The decision on the most appropriate procedure to provide to the patients the best healthcare possible is a critical and complex task in Intensive Care Units (ICU). Clinical Decision Support Systems (CDSS) should deal with huge amounts of data and online monitoring, analyzing numerous parameters and providing outputs in a short real-time. Although the advances attained in this area of knowledge new challenges should be taken into account in future CDSS developments, principally in ICUs environments. The next generation of CDSS will be pervasive and ubiquitous providing the doctors with the appropriate services and information in order to support decisions regardless the time or the local where they are. Consequently new requirements arise namely the privacy of data and the security in data access. This paper will present a pervasive perspective of the decision making process in the context of INTCare system, an intelligent decision support system for intensive medicine. Three scenarios are explored using data mining models continuously assessed and optimized. Some preliminary results are depicted and discussed.
\end{abstract}

Keywords: Real-time, Pervasive, Remotely access, Knowledge discovery in databases, Intensive care, INTCare, Intelligent decision support systems.

\section{Introduction}

Intensive care units (ICU) are a particular environment where a great amount of data related to the patients' condition is daily produced and collected. Physiological variables such as heart rate, blood pressure, temperature, ventilation and brain activity are constantly monitored on-line [1]. Due to the complex condition of critical patients and the huge amount of data, it can be hard for physicians to decide about the best procedure to provide them the best health care possible. The human factor can lead to errors in the decision making process; frequently, there is not enough time to analyse the situation because of stressful circumstances; furthermore, it is not possible to continuously analyse and memorize all the data [2] .

Care of the critically ill patients requires fast acquisition, registering and availability of data [3].

Accordingly, rapid interpretation of physiological time-series data and accurate assessment of patient state is crucial to patient monitoring in critical care. The data 
analysis allows supporting decision making through prediction and decision models. Algorithms that use Artificial Intelligence (AI) techniques have the potential to help achieve these tasks, but their development requires well- annotated patient data [4, 5].

We are deploying a real-time and situated intelligent decision support system, called INTCare ${ }^{1}$, whose main goal is to improve the health care, allowing the physicians to take a pro-active attitude in the patients' best interest [6,7].

INTCare is capable of predicting organ failure probability, the outcome of the patient for the next hour, as well as the best suited treatment to apply. To achieve this, it includes models induced by means of Data Mining (DM) techniques [6], [8-11].

Further improvements include the adjustment of the system to new requirements in order to make it pervasive and ubiquitous [12]. This allows the system to be universal, i.e. can be used anywhere and anytime, eliminating any sort of barrier be it time or place.

This paper is organized as follows. Section 2 presents some background related to Intelligent Decision Support Systems (IDSS), Knowledge Discovery in Databases (KDD), Intensive Medicine and the Pervasive Computing. In next sections the INTCare system is presented, focusing on its features (section 3), the information architecture (section 4), pervasive approach (section 5) and the latest DM models developed (section 6). Section 7 and 8 conclude this paper, presenting a discussion, a conclusion and pointing to future work.

\section{Background}

\subsection{Intelligent Decision Support Systems}

According to Turban [13], a Decision Support System (DSS) is an interactive, flexible and adaptable information system, developed to support a problem solution and to improve the decision making. These systems usually use AI techniques and are based on prediction and decision models that analyse a vast amount of variables to answer a question.

The decision making process can be divided in five phases: Intelligence, design, choice, implementation and monitoring [13]. Usually it is used in the development of rule based DSS [14]. However, these DSS are not adaptable to the environment in which they operate. To address this fault, Michalewicz [15] introduced the concept of Adaptive Business Intelligence (ABI). The main difference between this and a regular DSS is that it includes optimization that enables adaptability. An ABI system can be defined as "the discipline of using prediction and optimization techniques to build self-learning decisioning systems. ABI systems include elements of data mining, predictive modelling, forecasting, optimization, and adaptability, and are used to make better decisions." [15].

As it is known, predictive models' performance tends to degrade over time, so it is advantageous to include model re-evaluating on a regular basis so as to identify loss of accuracy [8] and enable their optimization. 
There is a particular type of DSS, the real-time DSS. Ideally, the later includes adaptive behaviour, supporting the decision making in real-time.

To achieve real-time DSS, there is a need for a continuous data monitoring and acquisition systems. It should also be able to update the models in real time without human intervention [6]. In medicine, most systems only use data monitoring to support its activities, without predictive behaviour and with poor integration with other clinical information.

\subsection{Knowledge Discovery from Databases}

$\mathrm{KDD}$ is one of the approaches used in Business Intelligence (BI). According to Negash [16], BI systems combine data gathering, data storage, and knowledge management with analytical tools to present complex and competitive information to planners and decision makers. KDD is an interactive and nontrivial process of extracting implicit and previously unknown and potentially useful and understandable information from data [17].

The KDD process is divided in 5 steps: Selection, pre-processing, transformation, data mining and interpretation/evaluation [18]. This process starts with raw data and ends with knowledge.

The automation of the knowledge acquisition process is desirable and it is achieved by using methods of several areas of expertise, like machine learning [9]. The knowledge acquisition takes advantage of KDD techniques, simplifying the process of decision support [8].

Knowledge discovery is a priority, constantly demanding for new, better suited efforts. Systems or tools capable of dealing with the steadily growing amount of data presented by information system, are in order [19].

\subsection{Intensive Medicine}

Intensive medicine can be defined as a multidisciplinary field of the medical sciences that deals with prevention, diagnosis and treatment of acute situations potentially reversible, in patients with failure of one or more vital functions [20].

These can be grouped into six organic systems: Liver, respiratory, cardiovascular, coagulation, central nervous and renal [21].

ICU are hospital services whose main goal is to provide health care to patients in critical situations and whose survival depends on the intensive care [22], [23].

In the ICU, the patients' vital signs are continuously monitored and their vital functions can be supported by medication or mechanical devices, until the patient is able to do it autonomously [22].

Clinical intervention is based on the degree of severity scores like the SOFA (Sequential Organ Failure Assessment) score, that allow the evaluation of the patient's condition according to a predefined set of values [24].

The assessment of these severity scores are based on several medical data acquired from bedside monitors, lab results and clinical records. 


\section{$2.4 \quad$ Real-Time}

A system that aims to support decision making must analyse many parameters and output in short real-time and consider online monitoring [25]. It is known that in the ICU setting, there is a huge amount of noisy, high dimensional numerical time series data describing patients. Consequently, such systems must go beyond classical medical knowledge acquisition, since they have to handle with high dimensional data in real-time.

Data acquisition in real-time implies the need for a system responsible for collecting the relevant data to the DSS. This process can be divided in two phases: monitoring and acquisition and storage. Initially, the required data (variables) for the project is identified for further being monitored by sensors or other technology. Subsequently, data is acquired and stored in DB.

This is a critical phase, for technical, human and environment factors are involved and may condition the quality of the data acquired by a gateway, for example, and its storage on a DB. Usually, the monitoring is continuous and there is a small percentage of failures. Although they may occur, they are relatively easy to correct. The biggest problem occurs in the communication between the monitoring system and the storage system.

In conclusion, monitoring in real-time is relatively easy; usually, problems arise in the data storage process.

\subsection{Pervasive Computing}

Satyanarayanan [26] characterizes the pervasive computing (ubiquitous) as an evolutionary step resulting from the harmonization of the fields of distributed computing and mobile computing (Fig. 1).

This involves not only issues related with saturated environments of communication and user interaction, but also in the support of user's mobility.

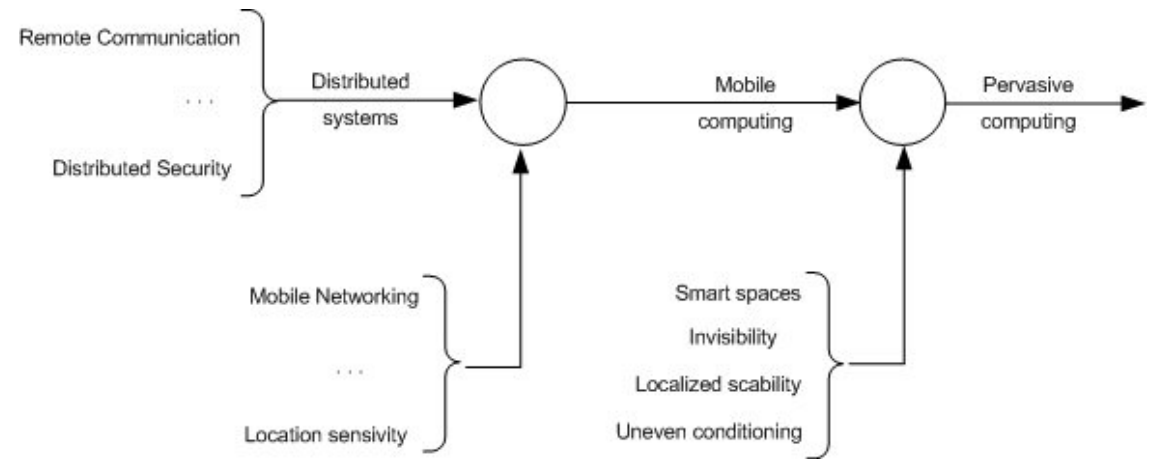

Fig. 1. Pervasive Computing [26] 
This type of computing has evolved with the emergence of the Internet. Nowadays, the Internet is easily accessible in all developed countries, schools, public organizations, at home, inside or outside of buildings.

The appearance and ease of access to wireless networks and communication systems contributed to its dissemination. The incorporation of computing devices, objects or sites for monitoring, allows a glimpse of real, physical, and enhanced with information and computing resources that can be used to facilitate human life in its various tasks (personal or social) or to improve business or organizational processes [27]. Complementarily, Banavar [27] also considers that pervasive computation can be:

(i) the way people think about and use mobile computing devices to perform tasks;

(ii) how applications are developed and implemented as form of support for these tasks;

(iii) How the environment is strengthened by the emergence and ubiquity of new information and features.

Almost all the concepts mentioned above can be migrated to pervasive health care. According to Varshney [28], Pervasive HealthCare (PH) can be defined as "health for everyone, anytime and anywhere by removing restrictions such as location and time, increasing both coverage and quality of healthcare". Such approach is essentially based on information that is stored and available online [29].

One of the targets of the system is to achieve the three points described above and to deliver medical information remotely and online through ubiquitous devices [31, 32].

Such approach contributes for the mitigation of medical errors caused by the lack of correct information at the place and time that it is required e.g., misdiagnosis. Decision errors may reach $50 \%$ of the total errors [33].

Ubiquity in electronic medical records enhances the analysis of the information by authorized users, anytime and anyplace [34] promoting a real time operation.

The granularity of time, the representation and retrieval of information play an important role in the mode as the diagnosis, prognosis and treatments are performed [35].

\section{The INTCare System}

INTCare is an IDSS for intensive medicine that is being developed in the ICU of the Hospital Santo António (HSA) in Porto, Portugal. It makes use of intelligent agents [36-38] that are capable of autonomous actions in order to meet its goals [7], [39].

To allow a pervasive access to the IDSS some also is essential take into account some requirements and concerns like privacy and security of the users, patients and system.

\subsection{System Features}

In order to model the information for KDD processing, the system attends some requirements: 
Online Learning - The system acts online, i.e., the DM models are induced using online data in opposition of an offline approach, where the data is gathered and processed afterwards;

Real-Time - The system actuates in real-time, for the data acquisition and storing is made immediately after the events take place to allow that decisions are taken whenever an event occurs;

Adaptability - The system has the ability to, automatically, optimize the models with new data when needed. This information is obtained from their evaluation results;

Data Mining Models - The success of IDSS depends, among others, on the acuity of the DM models, i.e., the prediction models must be reliable. These models make it possible to predict events and avert some clinical complications to the patients;

Decision Models - The achievement of the best solutions depend heavily on the decision models created. Those are based in factors like differentiation and decision that are applied on prediction models and can help the doctors to choose the better solution on the decision making process;

Optimization - The DM models are optimized over time. With this, their algorithms are in continuous training so that increasingly accurate and reliable solutions are returned, improving the models acuity;

Intelligent Agents - This type of agents makes the system work through autonomous actions that execute some essential tasks. Those tasks support some modules of the system: Data acquisition, data entry, knowledge management, inference and interface. The flexibility and efficiency of this kind of system emerges from the intelligent agents and their interaction [7].

Accuracy - The data available in the IDSS need to be accurate and reliable. The system need to have an autonomous mechanism to a pre-validation of the data. The final validation will be always done by a Human, normally by the nurse staff. This operation should be done on the ENR, moments after collection. With this, the user is sure that the data he can see online is guaranteed true.

Safety - All patient data should be safely stored in the database. The data security has to be ensured the access should be restricted. This is the one of the most critical aspects in this type of approach.

Pervasive / Ubiquitous - The system need to be prepared to work in ubiquitous devices like notebooks, PDAs and mobile phones. The internet plays an important role making the system available for users in anyplace. The ICU access policy should be available.

Privacy - There are two types of privacy: i) related to the patient and; ii) related to the health care professional. The patient identification should be always hidden to the people out of hospital. On the other hand the pieces of information recorded on this environment need to be identified and associated to one user, in order to find out responsibilities. Both types of identifications should be protected and masked.

Secure Access from Exterior - The hospital access point has to be protected from exterior connections and encrypted. A Virtual Private Network (VPN) with 
appropriate access protocols is a good option. Only people who have access to the ICU can see the information and operate, locally or remotely, with the IDSS. This system should implement a secure policy access and be prepared to work in a protected environment.

User Policy - The IDSS should include an inside (ICU environment) and an outside (remote connections) access policy, e.g. where and who can be consult or edit the data.

In order to accomplish the features presented above, some requirements should be considered:

- Fault tolerance capacities;

- Processing to remove null and noisy data;

- Automatic detection and processing of null patient identification;

- Automatic validation of the data taking into account the ranges (min, max) of each variable;

- Continuous data acquisition process;

- Time restrictions for the data acquisition and storage;

- Online learning mode;

- Digital data archive in order to promote the dematerialization of paper based processes (e.g., nursing records);

- Database extension to accommodate the data structures;

- Correct usage of the equipment that collects the vital signs.

\section{Information Architecture}

Patient management is supported by complex information systems, which brings the need for integration of the various types and sources of data [40].

In order to follow the requirements enumerated above, an information model was drawn, regarding the data acquisition module which includes three types of information sources:

- Bedside Monitors (BM);

- Lab Results (LR); and

- Electronic Nursing Records (ENR).

All sources can produce information to the system and that information can be used to develop predicting models in Intensive Care (knowledge). The development of an automated information system for ICU has to be in harmony with the whole information system and activities within the unit and the hospital [40].

The first type of sources relates to data acquisition from BM. This acquisition is in real-time, the data is received by a gateway, and it is stored on a DB table by an agent. Automatic acquisition eliminates transcription errors, improves the quality of records and allows the assembly of large electronic archives of vital sign data [40].

The second type of sources (LR) is the one that contains the less frequent observations, because the patient normally does this type of clinical analysis once or twice per day, except in extraordinary situations. With this method we can collect the 
data related with some clinical analysis, such as: number of blood platelets, creatinine, bilirubin, SOFA scores, partial pressure of oxygen in arterial blood and fraction of inspired oxygen.

\subsection{INTCare Sub-systems Functionality}

The INTCare System [6, 7] is divided into five subsystems: data acquisition, data entry, knowledge management, inference and interface. Fig 2 shows a model that is a part of INTCare system and represents an evolution of two subsystems: data acquisition and data entry. This subsystem is responsible for all activities of data acquisition and data store and will gather all required data into a data warehouse [4143]. The evolution of this architecture is prominent. Formerly, most of the data was registered in paper format, and it was necessary to manually put it in electronic format, i.e., the information was rarely stored in computers, except the information from the BM, which was automatically collected and stored in electronic format.

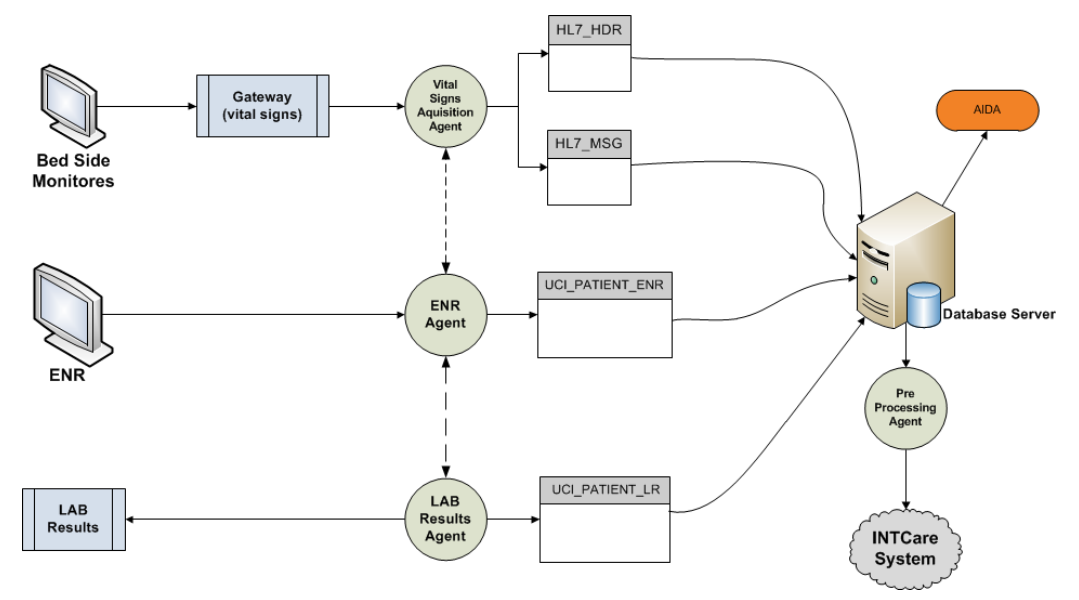

Fig. 2. The INTCare Data Acquisition Subsystem

The new architecture [41-43] contemplates the data acquisition from three sources and, regarding the information input, it is done either automatically (BM, LR) or automatically and manually (nursing records). The adjustment made to the system was the addition of one more data source and the creation of two more agents that enable storing information in the database (DB).

This modification is in course and it is the most important, because it makes possible the data acquisition in electronic and automatic mode for all data sources through multi-agent system. Whit this change, we will have all the necessary information in electronic format for the DM models and the decision support process, addressing the timing requirements of critical tasks.

\subsection{How These Subsystems Work}

The first type of data sources is the BM, which collects the patients' vital signs (VS). The gateway is connected to the monitors, reads the information and stores it on a DB 
through the data acquisition agent. This agent splits a HL7 [44] message in two, one with the header information and another with patient data.

The second source is the ENR [41]. It was developed with the objective of registering electronically the paper-based nursing records. With the ENR, the medical and nursing staff can register various types of data, like confirming if some therapeutic was performed or not, and they may consult all the present and past data about the patients. The last type of data sources is the LR, which is controlled by the clinical analyses agent that automatically stores all the LB from the patients.

All the data is stored in one DB and it can be accessed by the medical staff through a computer. The integrated data will be used by the INTCare system to create prevision and decision models.

The DM agent belongs to the sub-system knowledge management and it is in charge of retrieving the required data to feed the DM models and to train new models whenever their performance becomes unsatisfactory.

\section{Pervasive Approach}

One of the most important limitations in the ICU environment is related to the information availability which prevents the doctors to make the best decision for the patient. This is caused by the lack of integration of information in electronic format (a lot of records are still made in paper format).

Overcoming this limitation by means of a pervasive approach gives the doctors the possibility to review the data and act in the right time, i.e. before patient's clinical condition worsens, otherwise the doctor can only treat the patient when he is physically in the service.

In this context electronic medical records with detailed information about the patient may be analysed by anyone authorized, anytime and anywhere [34].

The information that returns from the applications is stored and made accessible from one single site. This implies some modifications to enable the access from small portable devices [29] (as defined in chapter 3).

Fig. 3 shows a pervasive perspective of the decision making process. The doctors can remotely, through a secure connection, consult the patient data or make a decision. This consult may have two purposes and can be done in real-time through the web platforms:

- ENR - shows all clinical data validated about the patient,

- INTCare - helps to make the decision based on some clinical predictions about the patient suggested by the Data Mining Engine.

The doctor's decision making process can start after the patient data is consulted or at the start, and can be made in a single fashion or discussed in a collaborative platform $[45,46]$.

After the decision be taken, this can be performed remotely, by a directly configuration in an ICU System or by giving some clinical instruction to the nurses. At the end, the performed decision will be used to optimize and adapt the decision and prevision models. 


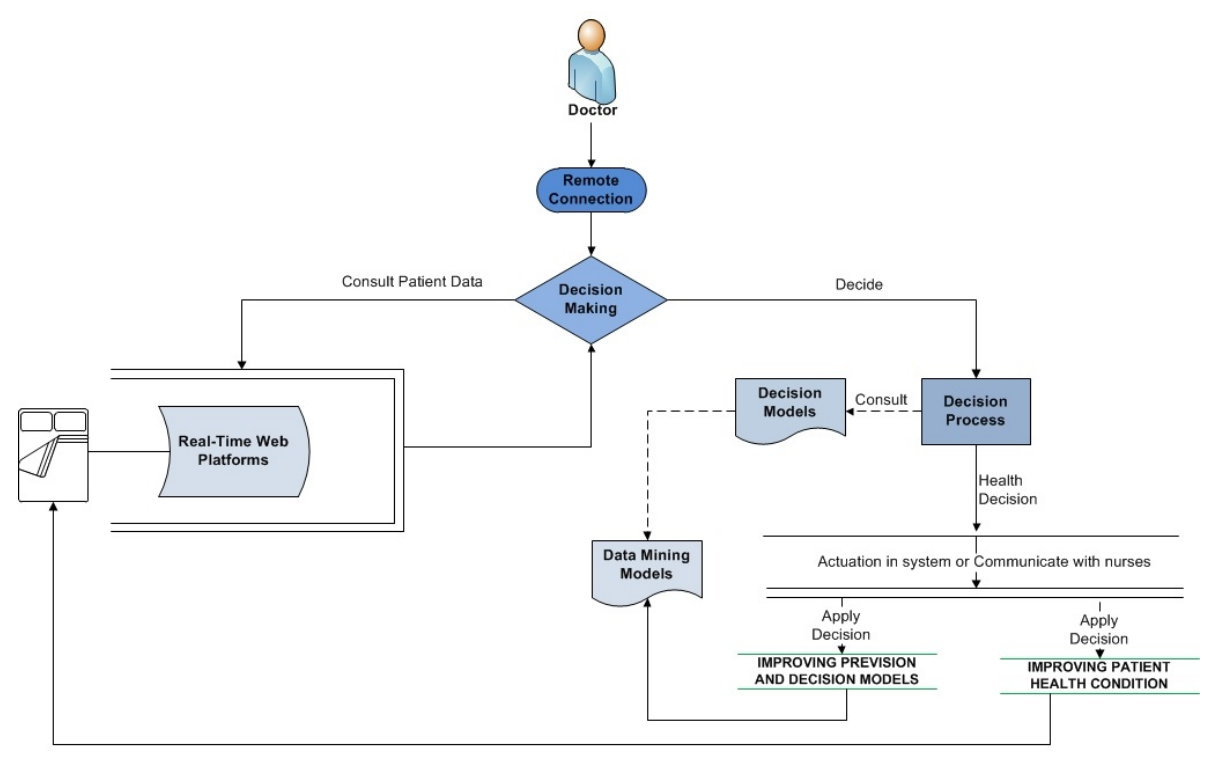

Fig. 3. Decision Support - Pervasive Approach

\section{Data Mining Models}

\subsection{Data Description}

The data used to generate the DM models originates from three distributed and heterogeneous sources: LR, BM and paper-based nursing records, presented and explained previously. Additionally, variables containing the case mix (information that remains unchanged during the patient's stay - age, admission from, admission type) were also considered. It was also included some calculated variables: Critical Events (CE), SOFA scores and a set of ratios relating the previous variables to the patients' length of stay.

The data was gathered in the ICU of HSA and it was collected in the first five days of stay of thirty two patients. The construction of the dataset was not automatically, the data from the LB and nursing records was manually registered, for the new adjustments of the system regarding the data acquisition and data entry were not developed at the time the models were generated.

\subsection{Features Selection}

For the prediction of the dysfunction/failure of each organic system and outcome, three scenarios were explored regarding the inclusion of the variables mentioned above - M1, M2, and M3 - where

M1 $=\{$ Hour, Case Mix, CE $\}$

$\mathrm{M} 2=\{$ Hour, Case Mix, CE, Ratios $\}$ 
M3 $=\{$ Hour, Case Mix, CE, SOFA $\}$.

For each model, the techniques applied were Artificial Neural Networks, Decision Trees, Regression and Ensemble methods.

\subsection{Results}

Table 1 shows the best results achieved for cardiovascular, respiratory, renal, liver, coagulation and neurological systems and outcome in terms of sensibility (i.e. percentage of failure and death correctly classified as such) as well as the scenario that produced the best results.

The models were developed for hourly prediction with the intent to make predictions as fast as possible, in the patients best interest.

Table 1. Sensibility of the DM models by system and outcome

\begin{tabular}{ccc}
\hline System & Sensibility $(\%)$ & Scenario \\
\hline Cardiovascular & 93.4 & M3 \\
Respiratory & 96.2 & M2 \\
Renal & 98.1 & M3 \\
Coagulation & 97.5 & M2 \\
\hline
\end{tabular}

\section{Discussion}

In this paper we presented the INTCare system, which is an IDSS for intensive medicine. It relies on the KDD process and AI algorithms to apply DM techniques for predicting outcomes that might support the course of action of doctors' decision.

Relying on intelligent agents, the system in divided into five sub-systems (data acquisition, data entry, knowledge management, inference and interface) that guarantee its functionality.

Since its beginning, INTCare has evolved towards using real-time and online clinical data so that the predictions can be as accurate and as soon as possible. As an IDSS, INTCare uses continuous data monitoring and acquisition systems that make possible for all information being available at the right time. This allows doctors to have a proactive attitude in patients' care.

The development of an ENR allows the integration of all necessary information regarding the patients' condition to be collected and integrated in just one application, which is a great gain in time and performance for the medical staff operating in the ICU. In addition to the patients' vital signs, data regarding their LR, procedures, medication, is also available by the time it is generated.

Moreover, the INTCare system is designed to address know issues of the ICU setting, such as noisy, high dimensional numerical time series data in real-time [25], as well as the data acquisition in real-time, storage, integration and rapid availability of all clinical information. The pervasive approach brought new features to the system 
enabling the remote access. However is necessary to reformulate the ICU environment taking into account some questions like privacy, security, connection and other concerns [34, 47-49].

\section{Conclusions and Future Work}

The main concern in ICU is to avoid or reverse organ failure, in order to preserve the patients' lives. The INTCare system is being developed for hourly prediction of the patients' clinical condition, i.e. the prediction of dysfunction/failure of the organ systems (cardiovascular, respiratory, renal, coagulation and liver systems) and outcome.

We believe that, with this fine grained prediction, it will be possible for the healthcare professionals to have a timely intervention and a proactive attitude, regardless the space where they are, so that worst complications for the patients may be avoided. A pervasive approach will have a strong impact. Context awareness is an important issue to adapt the applications to the current situation [45].

Further work will encompass the test of the DM models generated so far, with online and real-time data from the ICU of HSA, in order to guarantee their accuracy or, in case their performance decays, to optimize them. The models presented used data manually entered and the next step is to use them with the new adjustments of the system, i.e., online and in real-time. Prediction, optimization and adaptability are features that make INTCare an ABI system, whose maid goal it to allow the medical staff to make better decisions, at the right time and place, improving quality in health care. At the same time the pervasive approach will be tested and evaluated remotely.

The integration with the various data sources and with the rest information systems of the hospital has been supported by the development of an ENR and further related work include its test in the ICU and subsequently, its optimization.

Acknowledgements. The authors would like to thank FCT (Foundation of Science and Technology, Portugal) for the financial support through the contract PTDC/EIA/72819/2006.

\section{References}

1. Mahmoud, M.: Real-time data acquisition system for monitoring patients in intensive care unit. In: Multisensor, Multisource Information Fusion: Architectures, Algorithms and Applications, vol. 5090, pp. 320-326 (2003)

2. Pereira, M., et al.: Computer aided monitoring system of intensive care unit patients. WSEAS Transactions on Information Science and Applications 4, 78-84 (2007)

3. Gardner, R.M., et al.: Real time data acquisition: recommendations for the Medical Information Bus (MIB). International Journal of Clinical Monitoring and Computing 8, 251-258 (1991)

4. Ying, Z., et al.: Real-Time Evaluation of Patient Monitoring Algorithms for Critical Care at the Bedside. In: 29th Annual International Conference of the IEEE Engineering in Medicine and Biology Society, EMBS 2007, pp. 2783-2786 (2007) 
5. Morik, K.: Data analysis and knowledge validation in intensive care monitoring (2003)

6. Santos, M.F., et al.: Intelligent decision support in Intensive Care Medicine. In: 2nd International Conference on Knowledge Engineering and Decision Support, Lisbon, Portugal, pp. 401-405 (2006)

7. Gago, P., et al.: INTCare: a knowledge discovery based intelligent decision support system for intensive care medicine. Journal of Decision Systems (2006)

8. Gago, P., Santos, M.F.: Towards an Intelligent Decision Support System for Intensive Care Units. Presented at the 18th European Conference on Artificial Intelligence, Greece (2008)

9. Gago, P., et al.: Adaptive decision support for intensive care. In: 13th Portuguese Conference on Artificial Intelligence, Guimaraes, Portugal, pp. 415-425 (2007)

10. Silva, Á., et al.: Organ failure prediction based on clinical adverse events: a cluster model approach. In: 3rd International Conference on Artificial Intelligence and Applications (2003)

11. Silva, Á., et al.: Multiple organ failure diagnosis using adverse events and neural networks. In: 6th International Conference on Enterprise Information Systems, pp. 401-408 (2004)

12. Kwon, O., et al.: UbiDSS: a proactive intelligent decision support system as an expert system deploying ubiquitous computing technologies. Expert Systems with Applications 28, 149-161 (2005)

13. Turban, E., et al.: Decision Support Systems and Intelligent Systems, 7th edn. Prentice Hall (2005)

14. Arnott, D., Pervan, G.: A critical analysis of decision support systems research. In: Conference on Decision Support Systems, Prato, Italy, pp. 67-87 (2004)

15. Michalewicz, Z., et al.: Adaptive Business Intelligence. Springer, Heidelberg (2007)

16. Negash, S., Gray, P.: Business intelligence. Handbook on Decision Support Systems 2, 175-193

17. Frawley, W.J., et al.: Knowledge Discovery in Databases: An Overview. AI Magazine 13, 57-70 (1992)

18. Fayyad, U.M., et al.: From data mining to knowledge discovery: an overview (1996)

19. Lourenco, A., Belo, O.: Promoting agent-based knowledge discovery in medical intensive care units. WSEAS Transactions on Computers 2, 403-408 (2003)

20. Silva, A.: Modelos de Inteligência Artificial na análise da monitorização de eventos clínicos adversos, Disfunção/Falência de órgãos e prognóstico do doente critico. Tese de doutoramento, Ciências médicas, Universidade do Porto (2007)

21. Hall, J.B., et al.: Principles of Critical Care: McGraw-Hill's Access Medicine (2005)

22. Ramon, J., et al.: Mining data from intensive care patients. Advanced Engineering Informatics 21, 243-256 (2007)

23. Rao, S.M., Suhasini, T.: Organization of intensive care unit and predicting outcome of critical illness. Indian J. Anaesth. 47(5), 328-337 (2003)

24. Vincent, J.L., et al.: The SOFA (Sepsis-related Organ Failure Assessment) score to describe organ dysfunction/failure. Intensive Care Medicine 22, 707-710 (1996)

25. Morik, K., et al.: Combining statistical learning with a knowledge-based approach-a case study in intensive care monitoring, pp. 268-277

26. Satyanarayanan, M.: Pervasive computing: vision and challenges. IEEE Personal Communications 8, 10-17 (2002)

27. Banavar, G., et al.: Challenges: an application model for pervasive computing, p. 274 (2000)

28. Varshney, U.: Pervasive Healthcare. Computer 36, 138-140 (2003)

29. Mikkonen, M., et al.: User and concept studies as tools in developing mobile communication services for the elderly. Personal and Ubiquitous Computing 6, 113-124 (2002) 
30. Varshney, U.: Pervasive healthcare and wireless health monitoring. Mobile Networks and Applications 12, 113-127 (2007)

31. Dovey, S., Makeham, M., County, M., Kidd, M.: An international taxonomy for errors in general practice: a pilot study. The Medical Journal of Australia 177, 68-72 (2002)

32. Kohn, L.T., et al.: To Err Is Human: Building a Safer Health System. National Academy Press (2000)

33. Bergs, E.A.G., et al.: Communication during trauma resuscitation: do we know what is happening? Injury 36, 905-911 (2005)

34. Varshney, U.: Pervasive Healthcare Computing: EMR/EHR, Wireless and Health Monitoring. Springer-Verlag New York Inc. (2009)

35. Augusto, J.C.: Temporal reasoning for decision support in medicine. Artificial Intelligence in Medicine 33, 1-24 (2005)

36. Vilas-Boas, M., Portela, F., Santos, M.F., Machado, J., Abelha, A., Neves, J., Silva, A., Rua, F., Salazar, M., Quintas, C., Cabral, A.F.: Intelligent Decision Support in Intensive Care Units - Nursing Information Requirements

37. Abelha, A., et al.: Agency for Integration. Diffusion and Archive of Medical Information

38. Santos, M.F., et al.: INTCARE - Multi-agent approach for real-time Intelligent Decision Support in Intensive Medicine. Presented at the 3rd International Conference on Agents and Artificial Intelligence (ICAART), Rome, Italy (2011)

39. Jennings, N.R.: On agent-based software engineering. Artificial Intelligence 117, 277-296 (2000)

40. Fonseca, T., et al.: Vital Signs in Intensive Care: Automatic Acquisition and Consolidation into Electronic Patient Records. Journal of Medical Systems 33, 47-57 (2009)

41. Portela, F., Santos, M.F., Vilas-Boas, M., Machado, J., Abelha, A., Neves, J., Silva, A., Rua, F., Salazar, M., Quintas, C., Cabral, A.F.: Intelligent Decision Support in Intensive Care Units - Nursing Information Requirements. WSEAS Transactions on Informatics (2009)

42. Santos, M.F., Portela, F., Vilas-Boas, M., Machado, J., Abelha, A., Neves, J.: Information Architecture for Intelligent Decision Support in Intensive Medicine. Presented at the 8th WSEAS International Conference on Applied Computer \&amp; Applied Computational Science (ACACOS 2009), Hangzhou, China (2009)

43. Santos, M.F., et al.: Information Modeling for Real-Time Decision Support in Intensive Medicine. In: Chen, S.Y., Li, Q. (eds.) Proceedings of the 8th Wseas International Conference on Applied Computer and Applied Computational Science - Applied Computer and Applied Computational Science, pp. 360-365. World Scientific and Engineering Acad and Soc., Athens (2009)

44. Hooda, J.S., et al.: Health Level-7 compliant clinical patient records system, pp. 259-263

45. Villas Boas, M., et al.: Distributed and real time Data Mining in the Intensive Care Unit. Presented at the 19th European Conference on Artificial Intelligence - ECAI 2010, Lisbon, Portugal (2010)

46. Miranda, M., et al.: A group decision support system for staging of cancer. Electronic Healthcare 0001, 114-121 (2009)

47. Cook, D.J., Das, S.K.: Smart environments: technologies, protocols, and applications. Wiley-Interscience (2005)

48. Black, J.P., et al.: Pervasive Computing in Health Care: Smart Spaces and Enterprise Information Systems

49. O'Donoghue, J., Herbert, J., Sammon, D.: Patient Sensors: A Data Quality Perspective. In: Helal, S., Mitra, S., Wong, J., Chang, C.K., Mokhtari, M. (eds.) ICOST 2008. LNCS, vol. 5120, pp. 54-61. Springer, Heidelberg (2008) 